Italique $\quad \begin{aligned} & \text { Italique } \\ & \text { Poésie italienne de la Renaissance }\end{aligned}$

V | 2002

Varia

\title{
Indice degli autori e dei personaggi storici
}

(PDF)

\section{Corrado Bologna}

\section{(2) OpenEdition}

Journals

Edizione digitale

URL: http://journals.openedition.org/italique/154

DOI: 10.4000/italique.154

ISSN: 1663-4438

\section{Editore}

Librairie Droz

\section{Edizione cartacea}

Data di pubblicazione: 31 dicembre 2002

Paginazione: 141-155

ISBN: 2-600-00826-8

ISSN: 1423-3983

Notizia bibliografica digitale

Corrado Bologna, « Indice degli autori e dei personaggi storici », Italique [Online], V | 2002, online dal 06 octobre 2009, consultato il 19 avril 2019. URL : http://journals.openedition.org/italique/154; DOI :

10.4000/italique. 154

(c) Tous droits réservés 
I N D I C E D E I N O M I 



\section{INDICE DEGLI AUTORI E DEI PERSONAGGI STORICI ${ }^{\circledR}$}

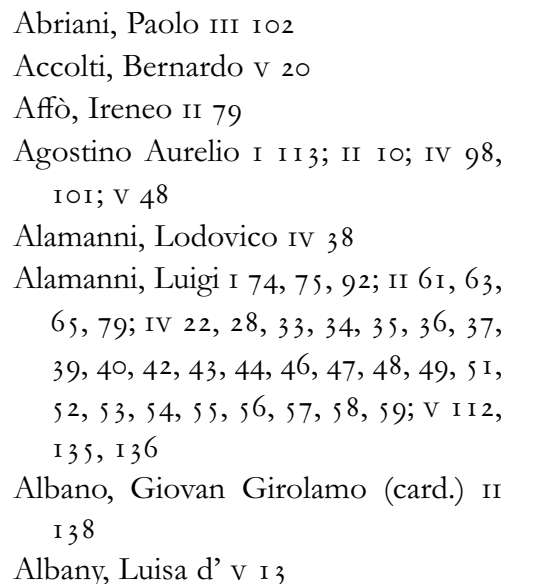

Alberti, Filippo III 47, 73

Alberti, Leon Battista IV I I

Albicante, Giovanni Alberto II 78

Albuzi, Aurelio II 99

Alciati, Andrea II 84, 86, 89, 90, 94, 97, 98, 99, I00, IOI; III 20, 2 I; V I I 5

Alcinoo V io6

Aldovrandi, Ulisse II I o०

Alençon, François d' I 83

Alessandro di Afrodisia II I 38

Alfani, Gianni I 28

Alfieri, Vittorio V I 3, 29

Alighieri, Dante I I 3, I4, 23, 30, 31, $8 \mathrm{I}, 82,83,84,85,86,87,88,89,90$, 9I , 92, 93; II 28, 29, IOO; III 28, 29,
Iо०, Iо6; IV 34, 35, 44, 45, 46, 47, 52,$53 ; \mathrm{V} \mathrm{I} 4, \mathrm{I} 5,39,57,58,62,63$, $70,74,84,86,87,89,9$ I, I 2 I

Allacci, Leone II 79

Almerici, Africano degli IV I 3

Almerici, Raniero degli IV 9, Iо, I 2, I 3, I 4, I6

Altan di Salvarolo, Federico v I 38

Altoviti, Bernardo IV 37

Amadei, Girolamo IV 68

Amalteo, Cornelio III 85

Amanio, Niccolò v I 7, 23

Amato, Raimondo I 5 I, 55, 58

Ambrogio II I 27, I 39, I 5 8, I 62

Ammirato, Scipione II 95, 97, IO2

Anacreonte II I 28, I 39

Ancina, Francesco III 85

Ancina, Giovenale III 85

Aneau, Barthélémy II 97; v 37

Angeli, Bonaventura v 30

Angiolieri, Cecco v 89

Antonio di Meglio II I I

Appiano Alessandrino IV 53

Aquino, Giacomo d' III 95

Aquino, Tommaso d' II I 52

Aragona, Giovanna d' I 4I, 52, 58, 72

Aragona, Maria d' I 58

Aragona, Tullia d' I 74

Ardizio, Curzio II 93, I I

I. I nomi, derivati da saggi redatti in più lingue, sono stati riportati alla forma italiana (lingua prevalente nell'uso della rivista) quando avesse largo corso: talché, ad esempio, sotto Francesco I re di Francia si trova anche quanto si riferisce a François ${ }^{\text {er }}$ roi de France e dizioni perifrastiche equivalenti; si rispetta invece la forma dell'originale in Hugues Capet e simili. Così si riducono alla forma italiana i nomi della classicità greca e latina. Per altro verso, si sono evitate designazioni ridondanti per indicare i personaggi, specie quelli con dignità dinastica, variamente nominati in saggi dovuti a più autori. Quando l'autore del saggio non ha indicato il nome (prénom) di un personaggio, nome che non si potesse ritrovare per altra via, si è aggiunta tra parentesi una qualifica professionale o altra, aggiunta ogni volta che è sembrato opportuno. Ciò vale naturalmente anche per l'altro Indice. $P . A$. 
Aretino, Pietro I 85, 87; II 37, 38, 40, 4I, 42, 5 I, 52; IV 65, 93; V I 2 I

Arigonio, Pietro III 85

Ariosto, Ludovico I 28, 41, 44, 70, 72,

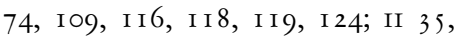
$37,38,39,40,41,42,43,44,45,46$, $47,48,49,50,5$ I $, 52,53,62,63$, I40, I4I; III 6O; IV 22, $23,25,28$, 56,57 ; V I6, I7, I $8,19,20,36$, I 6 , I 2 I

Aristotele I 73; II I 2I, I 25 , I 38, I4I, I62; V 100, 104, 106

Arlotti, Rodolfo II 89, 92, 99

Arnolfini, Vincenzo I 55

Arrigo VII di Lussemburgo imperatore I 88

Asinari, Federigo conte di Camerano III 74

Atanagi, Dionigi il 87, 88, 99; III 5 I, $52,54,58,59,60,63,7 \mathrm{I}, 79,80,82$

Atti, Isotta degli IV I I

Augurelli, Giovanni IV 34, 35

Augustini, Agostino III 95

Austria, Giovanni d' IV I I 3

Austria, Margherita d' IV 93

Auteuil, Moreau d' I 82

Àvalos, Alfonso d' I 4I; II 57

Àvalos, Ferdinando Francesco d' I 39, $40,54,56$

Àvalos, Francesco Ferdinando d' II 57

Àvalos Piccolomini, Costanza d' IV 79, 100

Avanzo, Ludovico II 99; III 5 I

Avogadro, Giulio v 4I

B. da Fano III 75

Bacchilide II 100

Badoer, Federico III 54

Bagnacavallo, Giovanni Battista v I 25

Baif, Jean-Antoine de v I 7, 26, 30

Baif, Lazare de I 84; v i I 8, I 24, I 34, I35

Baldini, Vittorio III 47, 69, 77

Balestrini, Nanni III Io6

Bandello, Matteo i 76; in 6o, 61, 62, 63
Barbati, Petronio v 39, 5 I

Barbieri, Giovanni Maria III 55, 8 I

Bargagli, Scipione II 97, 98

Barignano, Pietro IV 52

Barotti (prefatore di Tassoni) I I 23

Barozzi, Antonio I 65, 66, 69, 72, 73, $74,75,76$

Bartas, Guillaume de Saluste du II I45, I47, I49, I 50 , I 5 I, I 52 , I 53 , I55, I56, I57, I58, I59, I60, I6I, I62, I63, I64, I65

Bartoli, Girolamo II I 38

Basile, Giovan Battista I 30, 34, 49

Basilio di Cesarea II I 27, I 39, I 52 , I 53, I 56,162

pseudo-Basilio II I 59

Basini, Basinio II 22, 27

Baudelaire, Charles v I 24

Beccadelli, Ludovico IV 7 I

Beccuti, Francesco (il Coppetta) III I 2, 20, 75, 87; IV 92; V 23

Bellarmino, Roberto (card.) I 90, 94

Bellay, Joachim du V i I, I6, I7, I 8, I9, $20,21,22,25,26,28,30,31,35,36$, $37,38,39,40,4 \mathrm{I}, 42,43,44,45,46$, $47,48,50,51,52$

Belleau, Rémy I 82; v I4

Bellièvre, Claude v I 3

Bellori, Giovanni Pietro v I 25

Belloy, Pierre de I 85, 90

Beltrame, Camillo III 85

Bembo, Pietro I I I, I 2, I 3, I4, I 5 , I6, I7, I8, I9, 20, 22, 23, 24, 27, 28, 29, $30,3 \mathrm{I}, 32,33,37,39,4 \mathrm{I}, 43,53,74$, $76,84,97$; II 61 $63,86,87,88$, 95 ; III 20, 6I; IV 33, 34, 35, 52, 64, 66, 77, 93; V I4, I5, I6, 17, I8, I9, 22, $23,62,63,73,74,86,87,88,89,90$, 9I

Benalio, Giacomo III 84

Benalio, Giulio III 84, 8 5

Benci, Tommaso v 58

Bendidio, Lucrezia III 6o; IV 23

Benedetto da Mantova IV 98

Benedetto da Roma IV 99 
Benivieni, Girolamo III Iо, I 3, I 8; IV $34,35,52,53$

Bentivogli, Ercole IV 57

Bentivoglio famiglia IV 9

Bentivoglio, Alessandro in 58

Bentivoglio, Giovanni IV is

Bentivoglio, Violante iI 58, 60

Benvoglienti, Fabio v 39

Bernabei, Lazzaro IV is

Bernardini, Virginio is 96

Bernardino da Asti iv 66

Berni, Francesco I I 20, I 23 ; II 35, 36, 4I, 5I; V I 5

Bernini, Gian Lorenzo v I I I

Beroaldo, Filippo III I7, 2 I

Betti (libraio) I 87

Betussi, Giovanni III 78

Bevilacqua, Ernesto I I I 5

Bèze, Théodore de I 87, I 3

Bianchini, Scipione IV 7I

Bibbiena (Bernardo Dovizi da, card.) II $35,37,52$

Bindoni, Bernardino IV 98

Birago, Francesco IV 108, I09, I17, I I 8 , I I 9

Birague, René de (card.) I 92

Biralli, Simone II 96, 97

Blanchemain, Prosper v I4

Boccaccio, Giovanni I 28, 33, 59, 83, 86,87 ; II I 8, I9, 22, 27, 35, 37, 53; v $63,64,74,75$, I I 2

Bocchi, Achille II 97

Boiardo, Matteo Maria i 108, i 16, I 24; II I 2, I 8, I 9, 22, 24, 27, 42

Bonaccorsi (Biagio?) v 136

Bonagente, Annibale II 91, 92

Bonaventura da Bagnoregio IV 89, 90 , 99, IO I

Bonfigli, Luigi II I 37; IV I I 9

Bonifacio VIII (papa) I 85, 88

Borghesi, Antonio III 86

Borghesi, Diomede in 96; in 85

Borghini, Vincenzio v i I 6 , i 8

Borra, Luigi in 63, 79

Bourbon, Charles de (card.) I 82
Boverio, Zaccaria IV 63, 92, 94

Braccetti, Michele III 85

Bracci, Francesco (Cecchino) III 25, $26,27,28,29,30,31,32,33,34,35$, $36,37,38,39,40,4 \mathrm{I}, 42,44$

Bracciolini, Francesco III 99

Bragadino, Filippo III 83

Branchi, Baldo IV I6

Brignole Sale, Anton Giulio Iv 25

Britannico, Giovanni IV 57

Britonio, Girolamo IV 2I, 28

Brocardo, Antonio IV 5 I

Brucioli, Antonio IV 38, 39, 45, 46, 58

Bruni, Antonio III 95

Bruno, Giordano II I 40; V I 32

Bucer (Butzer), Martin I 87

Budé, Guillaume v I 22

Bugati, Gasparo II 78

Buonaccorsi, Giuliano IV 38, 39, 43

Buonanni, Vincenzo I 94

Buonarroti, Michelangelo I 43, 53, 72; II 38; III 25, 26, 27, 28, 29, 30, 3I, $33,34,35,36,37,38,39,40,4 \mathrm{I}, 43$, 44 ; IV 75 ; V I 27

Buondelmonti, Zanobi IV 53

Burchiello (Domenico di Giovanni, detto il) II 35, 5 I

Busdrago, Vincenzo I 50, 51, 55

Buzzati, Dino III Io I

Caburacci, Francesco II 97

Cacchi, Giuseppe I 49

Caccianemici, Francesco v i 25

Caetani, Enrico (card.) I 90

Caetano, Scipione III 92

Calcagnini, Celio V 35

Caliergi (stampatore) II ı $о 0$

Calmeta, Vincenzo III I 3, 20

Calogerà, Angelo v 79, 80, 89, 90, $9 \mathrm{I}$

Calogrosso, Gianotto IV I6

Calvin, Jean I 87; v 48

Calvino, Italo II 42

Calvo, Francesco v I 34

Camilli, Camillo II 97, Ioo 
Camillo Delminio, Giulio III 58, 82; IV 34; V I6, I9, ІІ 2, ІІ4, І 20, І 2 I, I 22, I 23, I 24, I 25, I 26, I 27, I 28 , I29, I30, I3I, I34, I35, I36, I37, I 38

Canale, Giovanni III ı I

Capaccio, Giulio Cesare II 97, Io0

Capello, Bernardo I 74

Capet, Hugues I 92

Capilupi, Lelio v I9, 20, 30

Capodagli, Giovanni Giuseppe v I 38

Caporali, Cesare III 47, 73

Cappel, Guillaume I 94

Cappel, Jacques I 90, 94

Cappello, Bernardo III 6I

Caproni, Giorgio III Ios

Caracciolo, Antonio IV 92

Caracciolo, Cola Antonio I 55

Caracciolo, Joan Francesco II 24, 27

Carafa, Cesare III 85

Carafa, Ferrante I 54, 58, 59

Cardona, Maria di I 100

Carducci, Giosue I 3 I, 34

Cariteo (Benedetto Gareth) I I I 3; II 63; III II I I 2, I 3, 20; IV 22, 24, 99, IOO; V I I

Carle, Lancelot de v 59, 72

Carlo di Valois (o piuttosto d'Angiò) re di Puglia I 88

Carlo V imperatore I I 5 , 4I; V 84

Carnesecchi, Maria IV 55

Carnesecchi, Pietro IV 73, 93, 96

Caro, Annibal I 74; II 89, 99; III 49, 50, $51,52,53,54,57,60,79,80 ;$ IV 63; V 23

Cartari, Vincenzo IV 28; v I I 4, I 38

Casanova, Marc'Antonio II 99

Cassola, Luigi cavalier v I 8

Castellani, Tommaso v 20, 2 I

Castelletti, Cristoforo III I

Castello, Bernardo IV i i 8

Castelvetro, Jacopo i 87

Castelvetro, Ludovico II I 3 I, I $37 ;$ V $79,80,8$ I $, 82,83,84,85,86,87,88$, 90,91
Castiglione, Baldassarre v 30, 63, 74

Castriota, Giovanna I 49

Cataneo, Maurizio II I62

Caterina da Siena I 84

Cattaneo, Cornelio (il Dormi) III 78

Cattaneo, Danese v i 30

Catullo, Gaio Valerio II 63, 64; IV 22, 53

Cavalcanti, Giovanni v 57

Cavalcanti, Guido I 83, 93; III I 8; v $57,58,62,63,64,70,73$

Caviceo, Jacopo v 63, 74

Cecco d'Ascoli (Francesco Stabili) II IO, I 2, I 5, I6, 20, 24, 27

Cenci, Giacomo III 52, 80

Cenne da la Chitarra III 79

Ceresara, Paride III 9, I I, I 5 , i 9; V i 6

Cervini, Marcello (card., poi papa Marcello II) IV 7 I, 95

Champier, Symphorien v 59, 60, 72, 73

Chappuys, Gabriel v 74

Chaucer, Geoffrey v i 2

Cheverny, Philippe Hurault de I 90

Cibo Varano, Caterina Iv 66, 94

Cicerone, Marco Tullio I 83; II I 53 ; III I7, 4I; V I 2 I

Cicogna, Emanuele II 57, 78

Cieco da Ferrara II 42

Cinna, Elvio II 86

Cino da Pistoia III 5 5; v 62, 87

Cinuzzi, Marco Antonio I 75

Ciotti, Giovan Battista II 162

Claudiano Claudio II I 3, 27, I 38

Clemente VII (papa) IV 47, 48, 49, 58

Coccapani, Carlo III 47, 48, 75

Coccio, Francesco Angelo v 20, 2 I

Colin, Jacques v 74

Colin, Nicolas I 82; v I4, I 5

Colonna, Giovanni (card.) I 55

Colonna, Marc'Antonio II 89

Colonna, Pompeo I 4I, 44

Colonna, Vittoria I I $5,23,38,39$, 40, $4 \mathrm{I}, 42,43,44,52,53,54,56,58,74$; III 5 I, 79; IV 21, 63, 64, 65, 66, 67, 
$68,69,71,73,74,75,76,78,79,80$, 81, 86, 88, 89, 91 92, 93, 94, 95, 96, 97, 99, 100; v 24

Colonna, Vittoria jr. I 52, 53

Comin da Trino (stampatore) I 38 ; II 96; IV 74; v 4I

Condé, Henri de I 84, 88

Contarini, Alessandro III 85

Contarini, Gasparo (card.) IV 67, 94, 95

Conti, Giusto de' II I 2, I 9, 28; IV I I, I 6

Contile, Luca II 58, 91, 92, 97, I I I III $62,63,68,86,87$

Corbinelli, Jacopo I 83, 84, 91, 93, 94; V I I 2

Corio, Bernardino III I 3

Cornaro, Federigo III 85

Cornazzano, Antonio IV I6

Corno, Giorgio II 95, 96

Correggio (Antonio Allegri, detto il) v I 28

Correggio, Isotta da V 4I

Correggio, Niccolò da III I 3, 20; V I 37

Corrozet, Gilles v 50

Corsi, Jacopo II 2 I

Corso, Rinaldo I 37, 38, 39, 40, 4I , 42, 43, 44; IV 75, 97, 99; V 4I

Cortese, Ersilia III 60

Costa, Lorenzo III I I; v I I 3

Costalius, Petrus II 97

Costanzi, Antonio III I 7, 2 I

Costanzo, Angelo di I 54, 63; II 99; v I I , 27

Cousin, Gilbert (Gilbertus Cognatus) V I 35

Cotta, Pomponio v i 26

Crenne, Helisenne de v 63, 74, 75

Crescimbeni, Giovanni Maria II 78; III 79,80

Crisolfo, Bernardino III 85

Cristina di Svezia v I 37

Cusano, Nicola IV 99

D’Alessandro, Guerino III Ios

Dal Pero, Giovanni Giacomo v 23
Daniello, Bernardino I 37; v 23, 25 , 39,90

D'Annunzio, Gabriele v i 13

Dal Pozzo, Cassiano v i i I, I 25 , I 28 , I 38

Dante da Maiano III 54, 55, 57

da Sabbio (stampatore) I 37

Dassy, François v 74

Davanzati, Chiaro II I 5, I6, 27

de Boy, Giovanni I 58

Degli Abati, Bocca II I 3 I

Degli Alberteschi, Mario Salamonio I 84

Degli Angioli, Nicolò III 75

De Jennaro, Pietro Jacopo IV 2 I

Dei Collenucci, Camilla IV I 3

Dei Collenucci, Matteo IV I 3

Dei Collenucci, Pandolfo IV I 3

Del Bene, Albizo Iv 38, 39, 43

Del Bene, Bartolomeo I 83

Delfin, Nicolò Iv 34

Dell'Abate, Giulio Camillo v I 30

Dell'Abate, Nicolò v I 25 , I 30

Della Casa, Giovanni I 59, 63, 74, 76, 97, 99; II 58, 86, 87, 88; V is

Della Macchia, Napoli (notaio) I 76

Della Palla, Battista Iv 53

Della Rovere, Francesco Maria III 85

Della Rovere, Giulia II I

Della Rovere, Guidobaldo III 62

Della Rovere, Isabella I 55

Della Torre, Francesco I 43

Della Valle, Francesco III 95

Del Garbo, Dino v 57, 62, 63, 73

Delio, Sebastiano V I 2 I

Del Riccio, Luigi in 26, 28, 30, 35, 36, 40, 43, 44

Del Rosso, Paolo iII 28

De' Mantelli, Giovanni (Tartaglia) IV 9, IO, I I, I4, I 5

Democrito II I 25

De' Pasti, Matteo IV I I

D'Erba Benedetto II 57

De' Ricci, Caterina IV 9I

De' Roberti, Ercole v I I 3 
De Rossi, cavalier (rimatore) III 76

Des Autels, Guillaume v 37

D'Esery, D. (bibliofilo) v I 3

Desportes, Philippe I 82; V I 2, I4, I 5 , I 7, 22, 23, 25, 26, 28, 30, 3 I, 35, 42, 52

Dietaiuti, Bondie II 24

Dionigi Areopagita IV 91, 99

Dolce, Ludovico I 59, 84, 8 5; II 97, 99, IOO; V I 5, I7, 22, 30, 39

Dolet, Étienne V I 2 I, I 34

Domenichi, Lodovico II 58 84, 85 , 97; III 78 ; V 25, 3 I

Domenichino (Domenico Zampieri, detto il) V I I

Doni, Anton Francesco III 78; V I 25 , I 26, I 27

Dorici, Luigi II I 40

Dorici, Valerio II I 40

Du Bouchet (storico) v I 3 , I9

Du Guillet, Pernette v 72

Du Jardin, Roland II I 45

Du Plessis-Mornay, Philippe I 86, 90, 94

Du Prat, famiglia I 82; v 29

Duret, Claude I 93

pseudo-Egidio Romano v 57, 62, 74

Enrico III re di Francia I 85

Enrico IV re di Francia e Navarra I $8 \mathrm{I}, 82,84,86,88,90,92$

Epicuro II I 48, I 56

Epicuro, Marc'Antonio II IO2; III 57

Equicola, Mario v 63, 74

Eraclito II I4 I

Erasmo da Rotterdam i 87; II I 39; v I07, I I 5, I 2 I, I 35

Erizzo, Sebastiano III 84

Erodoto II I 5,27

Este, Alfonso I d' II 5 I

Este, Ercole II d' IV 93, 94; V 79, I 34

Este, Ippolito d' (card.) IV 54

Este, Isabella d' II 39; III I I, I 9; IV 63; V I I 6

Estienne, Henri I I I 3; II I 39; v 22
Euripide v 36

Expilly, Claude v i 5

Fabre, François-Xavier v I 3

Falconetti, Camillo I 74

Fantauzzi, Giovanni IV is

Fanzino, Sigismondo IV 93

Farnese, Alessandro (card.) IV 68, 93, I I I

Farra, Alessandro II 9I, 97; III 76

Fauchet, Claude I 83

Fausto da Longiano, Sebastiano I 37; III 2 I; V I 2 I

Federico II Gonzaga marchese e poi duca di Mantova IV 99; v I 34

Fedro II IO, 27

Feliciano, Felice II I I

Felix, Alfius Flavianus III 42

Fenaruolo, Girolamo III 58, 62, 84, 8 5

Ferrier, Arnaud du I 86

Ferro, Giovanni II 91, 97, 98, 100, 102

Fiamma, Gabriele III 57, 82

Ficino, Marsilio III 9, I 3; V 48, 5 5, 56, $57,58,59,60,61,62,69,70,71,72$, $75,97,106$, I 32

Figliucci, Felice v 48

Figolino, Roberto III 85

Filelfo, Giovan Mario v 9I

Filippo I il Bello re di Francia I 85

Filippo II re di Spagna II 58, 78, Io I

Filosseno, Marcello II 24

Filostrato V I 25

Flaccius Illyricus, Matthias I 89, 90

Flaminio, Marc'Antonio IV 73, 89, 93, IOI; V I 2 I

Flaubert, Gustave III I $\circ 9$

Floncel, Albert-François v I 3

Flores, Juan de v $6_{3}$

Folengo, Teofilo (Merlin Cocai) I I07, I 20, I 24

Folgóre da Sangimignano III 79

Fortunio, Giovanni Francesco IV 34

Foscari, Girolamo I 23

Foxe, John I 89

Fracastoro, Girolamo IV 34; v 9I 
Francesco I re di Francia I 92; IV 33, $53,54,55$; V 59, I I 7, I 20, I 2 I, I 22 , I 24, I 28, I 29, I 30, I 3 I

Francesco I de' Medici granduca di Toscana V i I 6

Francesco I Sforza duca di Milano IV IO, I I, I 2

Francesco II Sforza duca di Milano II 58

Franco, Niccolò I 56, 57; IV 47, 49, 58, 93

Frangipani (rimatore) V I 5

Franzesi, Mattio III 79

Fregoso, Antonio Fileremo III I 3, I4, I $5,17,20,2$ I

Fregoso, Battista III I 3

Fregoso, Federico (card.) v 4I

Gabriele, Angelo I 23

Gabriele, Trifone I I 2, I 3, I4, 23; II 88; IV 34

Gabrielli, Pietro II 92

Gadda, Carlo Emilio III ıo6; v I I 3

Galieno, Claudio v 56

Galilei, Galileo IV I I 9

Galli, Angelo IV I 2, I7

Gallo, Filenio II Iо, 27

Gambara, Gian Francesco v 5 I

Gambara, Nicolò v 40, 5 I

Gambara, Veronica I I I, I 2, I 3, I4, I $5,16,17,18,19,20,23,24,38,4 \mathrm{I}$, 43; V 22, 24, 25, 26, 3I, 39, 40, 4I, $42,43,44,47,48,5$ I

Gambaro, Tommaso IV I 5 , I6

Garuffi, Giuseppe Malatesta II 97, 98

Gennari, Giovan Battista II 94, 95, IOI; III 78

Gentili, Scipio I 85; IV I I 8, I I 9

Gentillet, Innocent I 89

Gérard, Jean IV 70

Gesualdo, Carlo principe di Venosa II I 20, I 2 I

Gesualdo, Giovanni Andrea I 37; v I7, 19, 21, 30

Gheri, Cosimo IV 74
Giacomo da Lentini iI 24, 28

Giamblico v 106

Giannotti, Donato III 28, 30, 39, 40

Giberti, Giovan Matteo IV 66, 68

Giberti, Veronica IV 67

Giglioli, Tomaso I I 2 I, I 26

Giolito de' Ferrari (stampatore) I 49, 56, 63; II 58; V I 4, I 5, I 7, 22, 35, 36, $37,39,46,48$

Giorgione II 2I; V I I 8

Giotto V I I 3

Giovanni da Verona (fra' Giocondo) V I 22

Giovanni, Giovan Battista IV 49

Giovannini, Girolamo IV 58

Giovenale, Decimo Giunio IV 40, 43, $44,56,57$

Giovio, Giulio II 85

Giovio, Paolo I 4I; if 84, 85, 86, 89, 92, 97, 98, 99, 100, 102

Giraldi Cinzio, Giovan Battista III 83

Girardacci, Cherubino IV I 5

Giuliano l'Apostata I 92

Giulio Romano II 5 I; V I I I, I I6, I I 7, I I 8 , I 34

Giunta, Bernardo IV 34, 59

Giunti (stampatori) IV 35, 36, 47, 48, $49,50,52$

Giuseppe Flavio II I 38

Giusti (libraio) IV 49

Giustinian, Tommaso II 87

Giustiniano, Orsatto III 83, 8 5

Goethe, Johann Wolfgang von v I 17

Gondi famiglia I 82

Gondi, Bilicozzo IV 37

Gondi, Carlo III 44

Gonzaga, Agostino IV $6_{3}$

Gonzaga, Eleonora IV 67

Gonzaga, Ercole (card.) II Iоo; III 48, 62; IV 64, 66, 67, 92, 94

Gonzaga, Fabio II I 28

Gonzaga, Federico (vedi Federico II Gonzaga)

Gonzaga, Ferrante II 57, 58, 78; IV 63, 66, 94 
Gonzaga, Guglielmo (vedi Guglielmo Gonzaga)

Gonzaga, Ippolita I 75

Gonzaga, Isabella II 57, 59; IV 79

Gonzaga d'Àvalos, Isabella III 63

Gonzaga, Lucrezia III 48

Gonzaga di Novellara, Lucrezia v 40

Gonzaga, Margherita IV 93

Gonzaga, Scipione (card.) II 83, 84, $86,89,90,92,94,95$, I00, I 28; III 48,78

Gonzaga, Vincenzo (vedi Vincenzo I Gonzaga)

Goselini, Giuliano III 74

Goulart, Simon v 25

Gracián, Baltasar III 26, 39, 43

Gradenigo, Luigi II 92

Gradinico, Giorgio III 83

Gradinico Pietro III 83, 85

Grangier, Balthazar I 8I, 82

Grangier de Liverdis, Jean I 92

Granvelle, Antoine Perrenot de (card.) V I4

Grazzini, Anton Francesco (detto il Lasca) III 28, 29, 4I

Griffio, Giovan I 52

Grifolo, Giacomo I 74

Grillo, Angelo in 96; iII 74

Grizio, Paolo II 97

Grolier de Servières, Jean I 82

Groto, Luigi III 57, 82

Gruget, Claude v 74

Gryphe, Sebastien IV 33

Guadagni, Tommaso IV 38, 39

Gualengo, Giulio Cesare II I 34, I 40

Gualteruzzi, Carlo Iv 66, 74, Iо I

Gualtiero Anglico II Io

Guarini, Giovan Battista I 99, го0, IоI; II 89, 92, 95, IOO, IOI; III 47, 48, 75; IV 2I, 28; V 27

Guastavini, Giulio II I 24, I 38 ; IV I I 8, I 20

Guazzo, Stefano I 82

Guerrino (cioè Guarino Guarini) v 9I
Guglielmo Gonzaga duca di Mantova II IOI

Guicciardini, Francesco I 84; IV 48, 59

Guicciardini, Nicolò IV 48, 58

Guidetti, Francesco IV 34, 35, 52

Guidi, Benedetto III 52

Guidiccioni, Giovanni III 20, 68, 87; IV 63,$92 ; \mathrm{V} 2 \mathrm{I}$

Guise, Louis de Lorraine (card. de) I 82 ; V I

Guisone, Ferrante II I45, I46, I47, I49, I 50, I52, I53, I57, I60, I6I, I62, I64, I65

Haedus, Petrus III I 3

Hakluyt, Richard I 86

Henri (vedi Arrigo e Enrico)

Héroët, Antoine v 9, 55, 59, 63, 72

Hotman, François I 85, 90, 93

Iamblicus (vedi Giamblico)

Igino IV 23, 29

Ignazio di Loyola V I I 4, I 23, I 32, I 35

IsidorO II I 2, 24, 28

Jacopone da Todi I 84; II 24

Joyeuse, duc de v 26

Juste, François v 74

Labé, Louise v 63, 72

Laborde, Louis de V I I 7, I 33, I 38

La Gessée, Jean de I 83, 92

Lalli, Giovan Battista I I 2 I, I 26

Lambert, Constant III 99

Lancillotti (Tommasino de' Bianchi, detto) IV 65, 93

Landino, Cristoforo I 87, 88, 9I

Lando, Ortensio V 4I

Langeac, François de V I 2 I

Langeac, Jean de V I 2 I

L'Angelier, Abel v 23

L'Angelier, Arnoul v 50

La Porte, Maurice de V I 8

Larcara Spinola, Batina IV 54

Latini, Latino I 74, 75 
pseudo-Lattanzio II I 58

La Vallière, duc de v I 3

Le Fèvre de La Boderie, Guy I 83, 92, 93

Lemaire de Belges, Jean I 83; v I 7

Lenzi, Lorenzo III 6I

Leonardo da Vinci ir io, I5, I6, I7, 20, 24, 28; III 42

Leone X (papa) I I 5 ; II 35

Leone Ebreo v 63, 73, 74

Leopardi, Giacomo III 100

Lerme, Gabriel de II I 62

Lessing, Gotthold Ephraim III 26, 39

L'Estoile, Pierre de i 82, 85, 86, 87, 92

Ligeti, György III 90

Liruti, Giuseppe v I 38

Litolfi, Annibale II 58, 78

Litta, Pompeo II 78; IV I6, 55

Livio, Tito v I 25

Lomazzo, Gian Paolo II 78

Lorraine, Charles de (le Cardinal Vieux de) I 82; V I 5 , I 26

Lotti, Ottaviano IV 64, 93

Lotto, Lorenzo V i I 5 , I I 6

Lucano, Marco Anneo II II, I3, 20, 24, 28; IV I I I

Luciano di Samosata I I07, I 24; II I 36; IV 53

Lucinge, René de I 85, 93

Lucrezio Caro, Tito II I 24, I38, I48, I62; IV 22

Ludovico da Fossombrone iv 66, 67

Ludovico Sforza il Moro duca di Milano II 58; III I 3

Luther, Martin I 87, 88; V 4I

Llull, Ramon v I 32

Macedonio, Marcello III 97

Machiavelli, Niccolò I 76, 85, 89, 90; II I 2, 28, 35; IV 34, 42, 56, 59

Macrobio, Ambrogio Teodosio IV 22, I 20

Maggi, Giovan Battista II 57

Maggi, Ottaviano III 85

Maggs (libraio antiquario) v I4
Magno, Alessandro III 83

Magno, Celio III 58, 83

Magny, Olivier de V I 7, 20

Malatesta, Galeazzo IV I 2

Malatesta, Sigismondo IV 9, I I, I I, I 2

Malatesti, Malatesta IV I6

Malherbe, François de v I4

Malpiglio, Giovanlorenzo v 95, 96, 98, 99, 103,104

Malpiglio, Vincenzo v 95

Manarino, Giacomo (notaio) I 65, 76

Manfredi, Astorre IV I6

Manso, Giovan Battista in 96, I 45

Mantegna, Andrea III I I

Manuzio, Aldo I 37, 43; v 97, 106

Manzoni, Alessandro III ıоI; IV I I7, I 20

Marchesino, Francesco II 59, 64

Marco da Brescia Iv 69

Marescotti famiglia IV 9

Margherita di Valois regina di Navarra I 43; III 63; IV 73, 75; V 55, 59

Mariano Scolastico III Io, I 5

Marino, Giovan Battista I 97, 98, 99, IOO, IOI, I I 3 ; III IOO; IV 2I, 23, 24, 25, 26, 29; V I 24, I 25

Marot, Clément v I6, I7, 63

Marsuppini, Carlo ini 9

Marsuppini, Cristoforo v 57

Martelli, Lodovico IV 46

Martelli, Lorenzo IV 46

Martin, Jean v 9, 20, 55, 74

Martinengo, Marc'Antonio III 85

Martirano, Bernardino I 54, 58

Martirano, Coriolano I 66

Marzana, Camilla IV I 3, I 7

Marziale, Marco Valerio III 26; v 22

Marzio, Alessandro III 52, 80

Masières, Thibaut de II I 55, I64, I65

Massimi (card.) v I 25

Massimiliano I d'Austria II 58

Massini, Filippo III 97

Masson, Papire I 82, 84, 88

Matraini, Chiara I 74

Matteo (evangelista) I 42 
Mauro, Giovanni III 47, 73

Mazzuchelli, Gianmaria I 75; IV 58; v I 38

Mazzurelli, Cesare III 74

Mecenate, Gaio I 28

Medici famiglia IV 46, 47, 49, 57

Medici, Alessandro de' IV 48, 49, 59, 93

Medici, Caterina de' I 82; v 46

Medici, Cosimo de' IV 50

Medici, Francesco (vedi Francesco I de' Medici)

Medici, Giuliano de' I 44; III I I; IV 79

Medici, Ippolito de' (card.) I I 20; IV 63

Medici, Lorenzo de' II 24, 28, 35, 5 I; IV 2I; V 57, 62, 74

Melantone, Filippo (Philipp Schwarzerd) I 87

Melosio, Francesco III 96, 97

Mendoza, Diego Hurtado de v I 30

Meninni, Federico III I $о 0$

Mesmes, Jean-Pierre de II 5 I

Metaieri, Giannetto II I45

Metastasio, Pietro III 100

Metelli, Scipione III 73

Michiele (madrigalista) III 97

Milhaud, Darius III 99

Minturno, Antonio I 98, 99, Iо0

Mocenigo, Giacomo III 84

Mocenigo, Tommaso III 84

Molino, Girolamo III 54, 58, 80, 83, 86

Molino, Marco III 84, 8 5

Molza, Francesco Maria I 4I, 44, 74; v 23, 39

Monin, Jean-Édouard du II I45, I 62

Montaigne, Michel Eyquem de I 82; v IO2, 105,108

Montfaucon, Bernard de v I 38

Monti, Gerolamo II 78

Monti, Scipione de I 49

Monti, Vincenzo III 99

Montméja, Bernard de v 25

Montmorency, Anne de IV 73, 75

Mopso IV 35
Morone, Giovanni (card.) IV 65, 73, 79; v 89

Morosina (Faustina Morosina della Torre, detta la) I 32

Morselli, Guido III I I I

Morto da Feltre III 42

Mozzarello, Giovanni III 79; v I7, 2 I

Muratori, Ludovico Antonio v 79

Muret, Marc-Antoine de I 93; v I 8, I9, 22

Museo v 22

Muzio, Girolamo III 74; IV 22, 23, 69, $95 ; \mathrm{V} \mathrm{29}, \mathrm{I} 2 \mathrm{I}, \mathrm{I} 34$

Nacci, Francesco IV 46

Nannini, Remigio II 64

Navagero, Andrea III 58; V 9I

Navarre, Henri de (vedi Enrico IV)

Nelli, Giustiniano Iv 65

Nelli, Pietro IV 57

Nicolò III (papa) I 88

Nicolò IV (papa) I 83

Nicolò d'Aristotile (detto il Zoppino) I $38 ;$ IV $73,74,76$

Nigresoli, Antonio Mario v 23

Nostredame, Jean de I 83

Nuti, Giulio III 76

Nuvolara, Annibale di IV 38, 39, 55

Nyon (bibliotecario) v I 3

Ochino, Bernardino I 45; IV 63, 64, 65, $66,67,68,70,71,72,73,74,76,77$, $78,79,80,8$ I, 83, 89, 92, 93, 94, 95 , 98,99, I 00, Iо I

Olimpo da Sassoferrato II 24

Olivieri, Annibale IV I 2, I6, I7

Omero I I 24; IV Io9

Oporinus, Johann (libraio) I 88, 89

Orazio, Quinto Flacco I 28, 3 1, 32, 33 , 98; II I 3, 28; IV 24, 40, 57; V 44, 52

Ottieri, Ottiero III I05, I06, I07, 108, I IO

Ovidio, Publio Nasone I 29, 34, I I 2, II4, I 2 I; II II, I 5, 2I, 22, 23, 28, 79, 94, II 5, II6, I36, I64, I65; III 
I 7, 2I, 65, 67; IV 2I, 53, I 20; V I 8, I 25

Pagani, Galeotto III 85

Palazzi, Giovanni Andrea II 97

Paleotti, Gabriele v i i I

Pallavicino, Gaspare IV 79

Palma, Giovanni III 92

Palmario, Francesco IV 9, II, I 2, I4, I 5

Paoli, Pier Francesco III 95

Paolo di Tarso IV 92; V 24, 25, 42, 43, $47,48,52$

Paolo III (papa) III 58; IV 65, 66, 93

Paolucci, Alfonso II 5 I

Parabosco, Girolamo IV 22, 28; v 20, 22, 23

Paradin, Claude II 85, 97

Parini, Giuseppe III 102

Parise, Goffredo III IO I

Parronchi, Alessandro III Ios

Pas, Philippe de V 25, 3 I

Pascoli, Giovanni III Ios

Pasini (stampatore) IV 98

Pasolini, Pier Paolo in 106

Pasquati (stampatore) II 92

Pasquier, Etienne I 83, 88, 92, 94; v 22

Passero, Marc'Antonio I 5 5, 56, 59

Patrizi, Francesco III 63, 86

Peletier, Jacques v 20

Penni, Luca V I 25

Peperara, Laura IV 23

Perrot, Charles I 86

Perrot, François I 86, 87

Perrot, Milles II I 89, 93

Persio Flacco, Aulo IV 57

Perugino (Pietro Vannucci, detto il) III I I

Petrarca, Francesco I I I, I 2, I 3, I 4, I 5, I6, I 8, I9, 23, 28, 29, 30, 3 I, 33, $34,37,43,55,75,83,85,86,87,90$, I I 3 , I 25 ; II 24, 29, 30, 99, I 2 I, I 22 , I 24, I 3 I, I 36; III I7, 42, 5 I, 52, 53 , $56,57,79$, IO2; IV 2I, 34, 35, 4I, 52, 53, 54, 85, 97; V I I, I4, I 5, I6, I7,
I 8, I9, 20, 21, 22, 26, 36, 39, 6I, 62, $63,74,84,85,86,87,90$, I I 3 , г 2 I, I 34

Petrucci, Giovanni Antonio II 2 I, 29, 3 I

Peyrat, Jean du II I45, I62

Philieul, Vasquin v 52

Piantanida, Pietro II 58, 66, 78

Piccinino, Iacomo IV I 2

Piccolomini, Alessandro v I 4

Piccolomini, Francesco I 74

Picinelli, Filippo in 96, 98, гоo

Pico della Mirandola, Gianfrancesco v I03, I 108

Pico della Mirandola, Giovanni ini ıo, I 3, I 8, 20

Pietro da Cortona V I I I

Pighinucci, Martino I 55

Pignatelli, Ascanio II 89, 90

Pio II (papa) IV 9

Pio IX (papa) IV ıoo

Pio XII (papa) IV ıoo

Pio, Marco II I 28

Pindaro II 90, I00

Pirogallo, Filippo IV 74, 76

Pisani, Baldassarre III 97

Pisano, Ottavio II I 39

Pitagora V 106

Platina (Bartolomeo Sacchi, detto il) III I 3

Platone II 92; III I7, 5I; IV 53; V 48, $56,57,59,97,100,104,133$

Plauto, Tito Maccio II 37

Plinio, Gaio Secondo II I 5, 20, 24, 29, 99, I 54

Plutarco II I 27, I36, I38, I4I; V IoI, I07

Pole, Reginald iv 68, 7 I

Poliziano, Agnolo II I 3, 24, 29, 53, 6r, 63, 64; III I I; IV 35; V 57

Pontani, Filippo Maria III I 9

Pordenone (Giovanni Antonio de Sacchis, detto il) v i I 8

Porfirio V 106

Poro, Girolamo III 85 
Postel, Guillaume I 83, 84; V I 30 , I 37

Poupo, Pierre v 25, 32

Poussin, Nicolas V I I I, I 24, I 25 , I 30

Pratolini, Vasco III Io I

Primaticcio, Francesco V I17, I 8 , I 25 , I 26, I 27, I 29, I 3 I, I 34

Priuli, Daniel IV 73, 93

Proclo V 97, 98, 100, 103, 106

Properzio, Sesto Aurelio II 22, 29; III I7, 2I, 65; IV 35

Psello v io6

Pulci, Luigi II I 2, 24, 29, 42, 53

Pusterla, Giovan Francesco II 92

Quadrio, Francesco Saverio II 78, 79; III 79

Quattromani, Sertorio I 33

Quirini, Girolamo II 95

Quirini, Leonardo III 95

Rabelais, François v I I 5

Ragazzoni, Girolamo (card.) I 90, 94

Raimondi, Marc'Antonio II 5 I

Rainerio, Antonfrancesco IV 24; v I9, 30

Rainieri, Iacopo IV 63, 92

Rasse des Neux, François v I 3

Rebora, Clemente III 106

Recalcati, Ambrogio Iv 67, 93

Renata di Francia duchessa di Ferrara v 79

Reni, Guido V I I I

Ricci, Bartolomeo v 35

Richaud, Frédéric III 9I, 99

Ridolfi, Lucantonio I 82; III 5 I, 79

Rienzo, Gasparo II I45

Ripa, Cesare II I 37; V I I 4

Rizzardi, Felice v 24, 3 I

Robertet famiglia I 82

Roberto II re di Napoli v I I 3

Rodrigo, Joaquín III 99

Ronconi, Ludovico III 85

Ronsard, Pierre de I 83, 89; V I I, I4, I 5, I6, I7, I 8, I9, 20, 21, 22, 30, 35, $39,5 \mathrm{I}, 55,7 \mathrm{I}$
Rosa, Giovanni I 74

Rosci, Giovan Bernardo I 74

Rossi, Porzia de' III $6_{3}$

Rosso Fiorentino (Giovanni Battista di Iacopo de' Rossi, detto il) v i 7 , I I 8 , I 25 , I 26, I 28 , I 29, I 3 I, I 36

Rota, Berardino I 54, I00; III 66, 7I, 86

Rouillé, Guillaume I 82

Rovetti, Giovanni Andrea III 92

Rucellai famiglia I 82

Rucellai, Bernardo IV 34, 35

Rucellai, Cosimo IV 34, 35, 52

Ruscelli, Gerolamo I 37, 43, 5 2, 58, 59; II $85,90,98$, IOO, IOI; III 79, 82; IV 56,57 ; V I4, 2I, 25, $3 \mathrm{I}$

Rusticucci (card.) I 90

Sacchetti, Franco III 55

Sacchi, Andrea V I I I

Sacrati, Alberto IV 73, 75, 96

Saint-Gelais, Mellin de v I6, 20, 30

Saint-Sulpice, Antoine de v 29

Salel, Hugues v 36

Salutati, Coluccio III 43

Salvatorino, Giovan Giacopo III 79

Salvi, Virginia I 74; v 30

Salviani, Gaspare I Iо I, I06, I 20, I 22, I 24, I 25

Salviati, Francesco v I 30

Samperoli, Francesco IV I 3, I 7

Samperoli, Pacifica IV I 2, I 3

Sannazaro, Iacopo I 58; II 24, 29; III 65, 66, 7I, 86; IV 22, 34, 52, 53; v I 5,23

Sanseverina, Barbara III 47

Sansovino, Francesco III 63, 86; IV 57

Santini, Stefano II 89, 92, 98, Ior; III $47,48,50,59,75,78$

Sanvitale, Thiene Leonora II 93

Sanzio, Raffaello v i i I, i I 8

Sartre, Jean Paul III iog

Sasso, Panfilo II 9, I 3, I 5, I 7, 21, 24, 25, 29; V I 2

Sauguet, Henri (Henri Poupard) III 99 
Sauli famiglia V I 2 I

Sauli, Stefano V I 2 I

Savoia, Filiberta di I 44

Savoia, Luisa di IV 53

Savonarola, Girolamo i 84, 93; IV 40, 45

Savorgnan, Maria I 32

Scaligero, Giulio Cesare III 25, 26, 39

Scardente, Palmerio III 85

Scève, Maurice I 82; v 8, 9, I6, 55, 6I, $62,63,64,65,66,67,68,69,73,74$, 75

Scevola, Niccolò I 74

Scibec da Carpi, Francesco v i i 8

Scott, Walter III 9I, 99

Scotto, Giovanni Maria I 58

Sébillet, Thomas v 36, 37, 50

Secco, Nicolò II 78

Seghezzi, Anton Federigo I 30

Segni, Bardo IV 46, 52

Sellaio, Giacomo v 20

Selva, cavalier (rimatore) III 74, 75

Seneca, Lucio Anneo II I I, 29; IV 22

Senofonte III 9

Serafino Aquilano II 9, 21, 22, 27; IV 2I, 22, 23, 28; V II I I 2, I

Serassi, Pierantonio II 96; III 78

Serlio, Sebastiano v I 34

Sernini, Nino Iv 63, 66, 92

Serristora, Alessandra IV 38

Sertini, Tommaso IV 38

Servio, Mauro Onorato III I7; IV 29, I09, I I9, I 20

Sessa (stampatori) I 38,82

Sesto Empirico V IO2

Sforza, Alessandro IV I 2, I 3, I6

Sforza, Costanzo IV Io, I 2, I 3, I6, I 7

Sforza di Santa Fiora, Faustina II 58

Sforza, Francesco (vedi Francesco I Sforza e Francesco II Sforza)

Sforza, Giampaolo marchese di Caravaggio II 58

Sforza, Giovanni IV Io

Sforza, Guido Ascanio (card.) II 58

Sforza, Ippolita II 58
Sforza, Ludovico (vedi Ludovico Sforza)

Sforza, Massimiliano IV 38, 55

Sforza, Muzio I dei marchesi di Caravaggio II 57, 58, 66, 67, 68, 78

Sforza Bentivoglio, Ginevra IV 9, I I, I 3

Sigibaldi, Giovanni Domenico Iv 65, 93

Sigismondo Augusto di Polonia II 90

Sidney, Philip I 85

Silvestro II (papa) I 83

Silvius, Symon (Jean de la Haye) v 72

Simeoni, Gabriele II 85, 98

Simonetta, Bartolomeo III I 5

Simonide II Ioo

Sinesio V 106

Sisto V (papa) I $84,87,90$

Socrate V 58

Soderini, Giovanni IV 38, 39, 55

Soliani, Bartolomeo I I I 2

Soranzo, Vettor I I 4, I 5, 23, 34

Sorrentino (stampatore) II 5 I

Spannocchi, Giovan Francesco I 74

Speroni, Sperone I 84; II 90, I 39; III $48,58,74,82 ; \mathrm{V} \mathrm{37,} \mathrm{42,} \mathrm{51}, 63,73$, 74

Speusippo v 106

Spinola, Alessandro III 74

Spiriti (editore di Galeazzo di Tarsia nel i758) i 5 I, 54, 65

Stajano, Corrado III Io6

Stampa, Ermes III 92

Stampa, Gaspara v 24

Stampa, Massimiliano II 58

Stazio, Publio Papinio I 28; II 64, 79

Stefano Protonotaro II 24

Stigliani, Tommaso IV 26, 27

Stobeo V iо I, I08

Stocchi, Marco III 85

Stoppio, Niccolò III 85

Strabone II I 55

Strambone, Camillo I 56,57

Strozzi, Giovan Battista II 88; III 47, 74 
Strozzi, Giovan Maria IV 37, 55

Strozzi, Giovanni di Carlo III 29

Suetonio, Caio Tranquillo II 5 I; IV I I 8

Suganappo, Giovanni Paolo I 58

Taegio, Bartolomeo II 98; v I 26, I 35

Tamaro, Susanna III I 06

Tamisier, Pierre I 84, 93; v I4

Tansillo, Luigi I 54, 63, I00; v I 2, 23 , 27

Tarsia, Francesco di I 76

Tarsia, Galeazzo di I 49, 50, 5 I, 5 2, 53 , $54,55,56,57,58,59,63,64,65,66$, $67,69,70,71,72,73,74,75$

Tarsia, Juliella di I 75

Tassis, Ruggero de v I 4

Tasso, Bernardo I Iоo; il 59, 61, 63, 64, 79; III 54, 60, 63, 66, 67, 70, 71, $80,82,86$, Io6; IV 33, 5 I; V I 5

Tasso, Cristoforo II I 28

Tasso, Ercole II 96, 98, 99

Tasso, Torquato I 97, Iо0, Iог, I Iо, II 9, I 24; II 38, 39, 52, 84, 85, 86, $87,88,89,93,95,96,97,98$, гог,

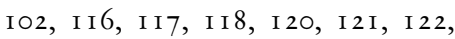
I 24, I 25, I 26, I 27, I 28, I 29, I 3 I, I 32, I33, I36, I37, I38, I39, I40, I4I, I45, I46, I47, I48, I49, I 50, I 5 I, I 52 , I53, I 54, I 55, I 56, I 57 , I 58 , I 59, I60, I6I, I62, I63, I64, I65; III 47, 48, 59, 6I, 63, 67, 7 I, 73, $74,76,77,78,79,86$, го5, го6, 108, I09; IV 23, 25, 26, 27, 29, I05, I08, III, II 2, II 3 , II4, II , II6, II 7 , II 8, II9, I 20, I 2I; V I 5, 21, 27, 95 , $96,97,98,99$, гог, го2, 103, 104, 105, $106,107,108$, I 30

Tassoni, Alessandro i 105, го6, 107, II 2, II 5 , II7, II 8, II9, I24, I25, I 26

Tebaldeo, Antonio I 72, 76; ir 9, 31, 6I; V I I, I 4, 27

Telesio, Antonio III Is

Teocrito IV 53; V 22

Teognide II I 27
Terenzio, Publio II 37, 52

Terracina, Laura I 49, 50, 5 I, 55, 56

Terracina, Polidoro I 55

Terminio, Antonio I 55, 56, 59

Thou, Jacques-Auguste de I 82, 8 , , 86, 93; v I 4

Tibullo, Albio II I I, 29; IV 53

Tiepolo, Giambattista v I I6, I 1 7, I 33

Tiepolo, Giandomenico v i I6, I 7

Tintoretto (Jacopo Robusti, detto il) v I 7

Tiraboschi, Girolamo III 80; v 82, 89

Tiron, abbé de (Philippe Desportes) v 22

Toledo, don Pedro di I 59, 65, 66

Toledo, Garcia di I 53, 59

Tolomei, Claudio I 74; III 52, 58, 80

Tolomei, Lattanzio I 74

Tomitano, Bernardino v 20, 37, 38

Tommaso III marchese di Saluzzo v I I 5

Tortoletti, Bartolomeo III 102

Toscanella, Orazio v I 25 , I 26

Tosco, Annibale v 22

Tournes, Jean de i 82; v i6, 61, 62, 74

Trini, Pietro III 47

Trissino, Gian Giorgio II I 3 I, I 40; IV $33,34,35,51,52$

Tritonio, Ruggero III 85

Trivulzio, Agostino (card.) IV 67, 94

Tura, Cosmé v i I 3

Turamino, Virgilio III 75

Tyard, Pontus de V I I, I 5, I 9, 5 5, 74

Ubaldini, Petruccio I 85,87

Uberti, Fazio degli II 24, 27; III 79

Uc de Saint-Circ I 28, 29, 33

Ungaretti, Giuseppe III Ios

Utinense, Alberto v 44, 52

Urbano VIII (papa) v i I I

Urfé, Honoré d'v i 5,29

Valdés, Juan de IV 73, 96, 98

Valentini, Filippo v 79, 80, 81, 82, 83 , $84,87,88,89,90,91,92$ 
Valeriano, Giovanni in 98

Valgrisi, Vincenzo IV 75, 76

Valla, Lorenzo III 9

Valois, casa di I 8 I, 83

Valvasone, Erasmo di IV 24, 25,29

Vandelli, Domenico v 89

Varano, Ercole III 47, 75

Varchi, Benedetto I 82, 84; II 86, 87, 88, 99; III 28, 40, 50, 6I, 79, 86

Vario, Rufo Lucio in 86

Vasari, Giorgio III 28, 29, 4I, 42

Vecellio, Tiziano III 85 ; IV 79; V II 7, II 8, I 2I, I 30

Vega, Garcilaso de la I ı०o, Iо I

Velásquez, Diego Rodríguez da Silva y V I I I

Vellutello, Alessandro I 37, 38, 39, 40, 43,44

Vendramini, Giovanni II 57, 58, 59, 60, 61, 62, 63, 64, 66, 67, 68, 78

Veniero, Domenico II 58; III 52, 54, $55,56,57,58,8$ I $, 83,85$

Veniero, Luigi III 83

Veniero, Marco III 84

Verdier, Antoine du I 83, 92

Verdizzotti, Giovan Mario III 58, 8 5

Verghicci, Hostilio III 85

Viani, Fulvio III 60

Vida, Girolamo I I 25; v 9I
Vieira, António v I 37

Villani, Nicola I I 2 I, I 25

Vincenzo I Gonzaga duca di Mantova II 93, I45, I46, I62; IV IO5

Vinciguerra, Antonio IV 57

Viotti, Seth (stampatore) III 47

Virgilio, Publio Marone I 28, 29, 83 , I 2 I; II II I I 3, I9, 22, $25,30,62,79$, $85,86,92$, I 37, I 39, I64; IV 22, 53,

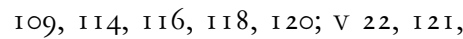
I 25

Visconti, Annibale II 59

Visconti, Gasparo III I 3, 20

Vitale, Giovanni Battista V 25, 3 I

Vittorini, Elio III Io I

Volponi, Paolo III Io6

Wolfe, John I 85,87

Zabata, Cristoforo III 69, 74

Zane, Giacomo III 58, 8 5

Zanzotto, Andrea ini io5, io6

Zeno, Apostolo II 57, 78; III 80; IV 58

Zito, Vincenzo III 95

Zorzi, Francesco v I I 2, I 23, I 30, I 35 , I 37

Zucco, Accio II Io

Zwichem, Viglius V I 2 I, I 22, I 35

Zwingli, Ulrich I 87 